\title{
An experimental proposal for low order laboratory animals' extension of metabolic life
}

\author{
Mario Gosalvez \\ Clínica and Hospital Puerta de Hierro, Madrid, Spain \\ Email: dmg.secre@gmail.com
}

Received 6 September 2013; revised 18 October 2013; accepted 1 November 2013

Copyright (c) 2013 Mario Gosalvez. This is an open access article distributed under the Creative Commons Attribution License, which permits unrestricted use, distribution, and reproduction in any medium, provided the original work is properly cited.

\begin{abstract}
Essential bibliography, with therein references included, is presented owing to the contribution of the author groups to Mitochondrial Filamentation, which is a new emerging field of physiological energy metabolism. These studies provide the first seed concept for trials to extend the metabolic life, for a few days, in low order laboratory mammals killed by electrocution, as a first type of accidental death. It is proposed, essentially, to cool out the corpses very soon after death at $12^{\circ} \mathrm{C}-14^{\circ} \mathrm{C}$ and take advantage of the effect super magnetism to counteract the force of gravity to install a net recurrent cycle of oxygen consumption and oxygen production by filamented mitochondria in all the organism tissues. Once the cause of death had been corrected adequately, it is possible to try the reanimation to experience the full life of the corpse with highly sophisticated methodology.
\end{abstract}

Keywords: Metabolic Life; Transitory Physiological Death; Cooling of Corpses; Super Magnetism;

Mitochondrial Filamentation; Clinical and

Experimental Resuscitation Techniques

\section{INTRODUCTION}

I would like to draw your attention to a possible transient state of death. I refer to physiological death, when the heart stops beating, the blood no longer circulates and settles and the EEG readings are flat. Depending on body temperature after death, the duration in a state of metabolic life will vary and complete reanimation is possible by means of greater or lesser sophistication in a highly advanced hospital if the state of death has not lasted longer than approximately two hours.

I ventured to suggest in Japan in 2008 [1] that we could extend the duration of metabolic life for those pos-

*Head, Experimental Biochemistry (1970-2010). sible transient corpses for more or less days, as long as we took into account the wonderful capabilities of filamented cellular mitochondria. We would then be able to bring back many physiologically dead animals to life for a few days, or even weeks, although, of course, in such circumstances, they would suffer a significant loss of body weight if more than three weeks in this special physiological death cold transitory slow metabolic period.

We suggest that studies be carried out on laboratory mammals which, if successful, could possibly be extended to babies who suffer infant death syndrome. With some more years elapsing, if it is feasible, we could widen the scope to include adults also with the adequate legal and insurance safeguards. This could drive medicine on that one step further with medical and surgical capabilities uniting to vanquish physiological death in a much greater number of cases than at present. In my humble opinion for full complex organisms, deep freezing cryogenics cannot afford lasting too longer coming back to physiological life.

Nature with the different ways of hibernation has solved the problem of suspending active life in very low temperature slowing down metabolic activity. As I will spell out that in the following text, my proposal is just based on extending the time of metabolic time for as long could be possible after physiological transitory death.

\section{MITOCHONDRIAL FILAMENTATION AND THE FORCE OF GRAVITY}

Our group has contributed to a greater physiological understanding of mitochondria, the cellular organelles with double membrane in which cellular respiration takes place associated with synthesis of adenosine triphosphate which is the basic unit of energy metabolism.

Using a method of mitochondrial isolation with less mechanical, thermal and chemical trauma, we discovered, above all, that when the animal is at rest, that the mitochondria are recursively filamented radially in their outer 
membrane by short filaments 25, 10 and 5 nanometres thick. In hypoxia, filamented mitochondria produce a greater amount of oxygen than they use up with bicarbonate present. Recent reviews of this emerging field and its possible future applications are published openly on line [2-8]. The references there in are sufficient to explain our expertise in this new field of the physiological bionergetics.

The net oxygen production by the filamented mitochondria is achieved in hypoxia in the presence of a medium which fundamentally mimics the cytoplasm. In hyperoxia, the oxygen consumption of those mitochondria is much greater than the oxygen produced. Keeping the oxygen concentration "in vitro", at the physiological level, an oscillation between oxygen consumption and production can be achieved. If this oscillation could be obtained "in vivo" at $14^{\circ} \mathrm{C}$ it would probably lead to the cellular metabolism being maintained for longer periods. In my present opinion one month could be the limit in organisms at their normality range in body weight.

We dare to think, perhaps, that by installing in the laboratory animals, changed into transient corpses using methods which simulate accidents, (I think first in artificial electrocution by high voltages), a consumptionproduction oscillation of oxygen by filamented mitochondria of their tissues, the extension of metabolic life could be achieved if everything is done right from the outset at $14^{\circ} \mathrm{C}$.

This could possibly achieve the extension of the metabolic life period of the animal corpse for many hours. After even a few days in that state perhaps a complete reanimation could be attempted using state-of-the art clinical methods in the field of anaesthetic resuscitation.

How might that chronic oscillation of oxygen consumption-production be induced into the tissues of young rabbits sacrificed by reversible accidents in order to extend the time which the corpse can be maintained with an active metabolic rate?

I think that by submitting the animal to very intense magnetic fields with different polarities, negative or positive. Or, in other words, by simulating a decrease or increase in the force of gravity. The simulation of a decrease in the force of gravity has already been tried on rats, by levitating them in the cavity of a super-magnet. Levitation is one of the things people like most about super-magnetism. It is based on the simulation of decreasing the force of gravity by means of the counteraction by very strong magnetic fields of the force of attraction of the Earth's mass on the subject.

A decrease in the force of gravity increases mitochondrial filamentation and decreases motor activity. The opposite occurs when the force of gravity is increased: motor activity increases and mitochondrial filamentation decreases. An increase of mitochondrial filamentation raises oxygen production and a decrease of mitochondrial filamentation raises the amount of oxygen consumed. We have observed these phenomena from measurements on filamented mitochondrial on different motor activities with different estimated values of the vertical gravity exercised on Madrid. This was published in a internal report of Spain's National Centre of Medical and Surgical research. The reasons for this dependency that we proposed could be related through a concomitance of mitochondrial filamentation-defilamentation-recurrent cycles, of all the organism tissues with body number of movements. This concomitance relationship that we proposed perhaps would be regulated by the light variations and other irradiation and magnetic cycles of the pineal gland.

A negative-positive oscillatory cycle would therefore have to be installed in the super-magnet, leading to the simulation of a recurrent, chronic increase and decrease of the force of gravity on the animal placed in a cold bag in the cavity in which the desired magnetic changes are created.

This is now possible in the United States of America, Japan and Germany, for instance, where the largest super-magnets are found. But only on small mammals; doubtless the smallest rabbits, recently weaned, would fit into the largest super-magnet currently available. But, for larger animals, such as a young sheep or a young mare, it would neither be possible nor practicable to attempt it, using that method of creating very strong magnetic fields which are based on the manufacturing of huge solenoids.

Now is not the time or indeed the place, to articulate my thinking on other ways of making strong magnetic fields with much less bulky equipment capable of simulating changes in the force of gravity in a large cavity. However, it will suffice just to add that I believe that different, inexpensive, ways can be tried out quite quickly. Specially those based in our in invention of megaelectric accumulators copying artificially mito- chondria (generic patent pending) geared to circulate electricity instead to electric power accumulation.

\section{COOLING OF THE TRANSITORY CORPSE}

Following the not irreversible electrocution, as a first example of "accidental death", the interior spaces of the animal corpse would have to be filled with suitable fluids: the abdominal and pleural cavities, the complete alimentary canal, etc. Then the corpse, duly sutured and shaved, would be placed as a liquid block in a liquid bag at $14^{\circ} \mathrm{C}$.

The composition of the internal and external fluids would be a matter for experts in physiological biochemistry, but following a due trial and error process, with the correct molecular nutritional and diffusion facilitator additives, the predominant solids, liquids and gases in 
the makeup of these fluids could be listed sufficiently accurately, in order to successfully attempt to maintain the active metabolism at $14^{\circ} \mathrm{C}$.

At that temperature the membranes would not disintegrate, the cellular skeleton of contractile proteins, the cytoskeleton, would be intact and, above all, as long as enough intercellular oxygen is always present, the hydrolytic enzymes for proteins, lipids and carbohydrates of the cytoplasmic vesicles would not be released. This is what I think is essentially responsible for the necrosis or putrification of tissues when there is insufficient oxygen supply due to a deficiency of the blood circulation destabilizing the membranes of the lysosomes.

\section{LIQUID BLOCK ELECTROPHORESIS}

The cold bag, with the animal corpse in a liquid block, would rotate slowly on its longitudinal axis in consonance with the super-magnetic oscillation. This will allow the low molecular weight components, with a negative or positive charge, to alternatively pass through the membrane pores of the cellular tissues.

This point is also susceptible to trial and error, supplementing it appropriately with membrane stabilizers and electrophoretic diffusion modulators of one or other sign. Evidently there would be the limitations, at the beginning of the experimentation, of the size of the cavity in which the strong magnetic fields are generated. When we reach a capability to experiment on larger animals this aspect could be addressed with the utmost perfection, especially when possible on the tailed primate.

My contribution, as a consultant, in this endeavor, which in some years' time could perhaps assume a major international dimension, would fundamentally be in the subsequent stage: the formation in the animal corpse, in a liquid block, of a production-consumption oscillation of oxygen by the mitochondria. I have a great familiarity, always accompanied by an enormous respect, for those tiny dancers of biological energy, whether they are naked, or preferably more or less clothed with contractile filaments.

\section{MAGNETIC OSCILLATION AND INTRACELLULAR OXYGEN}

I will try to pull together as concisely as possible what my knowledge on mitochondria is, now that perhaps the first stuttering new steps forward are being taken for life in our society. I would say first of all that our science is at such an early stage that what we know about filamented mitochondria and their possible concatenation with all the biological cycles is but a drop in the ocean.

The amplitude of the magnetic oscillation needed is in no way as great as that needed to levitate or squash a rat. We need a magnetic oscillation which can simulate varia- tions in vertical gravity on the subject of the experiment, ranging from the strongest to the weakest gravity force known for any point on the planet. That is not difficult, but for large animals, solenoids, huge magnetic coils, will not be enough.

In addition to their amplitude the wavelength of the oscillation should be precisely determined. To this end, we could start with perfused organs, especially the liver and lungs, at $14^{\circ} \mathrm{C}$, with a perfect monitoring system on oxygen concentration, carbon dioxide, hydrogen ions, potassium and sodium, at least both intracellular as well as extracellular, during the period of oscillation. That would enable the sequential destruction of the samples in order to determine by electron microscopy the variable degree of mitochondrial filamentation for each simulated stimulated or decreased force of gravity. With that in each organ, passing later to the laboratory animals of different sizes, the required evaluation could be made of the possible gravitational "tuning fork", for the mitochondrial concatenation of all the physiological tissues of the particularly chosen transitorily dead organism in the experiment. In my humble opinion that concatenating fork would reside in the "Triad Epiphysis-Hypotha- lamic-Pituitary" possible mitochondrial inter relation system,

When that point has been reached, the temperature would need to be gradually increased while the magnetic oscillation as well as the corresponding oscillation of oxygen production and consumption flattened out, until reaching, after following all the required steps in order, the moment to awaken the animal from its deep slumber.

\section{REANIMATION}

In my modest opinion the critical point at which to begin to wake up the superior animal, whose most intelligent behavioral patterns would have been known before its death, would occur when normal body temperature had been maintained for 24 hours. On that day the oscillation of oxygen production-consumption would be more or less flat, depending on the nature of the experimental subject. Almost daily weight loss due to the consumption of endogenous nutrients would be faster owing to the rate of metabolic activity, close to a temperature of $37^{\circ} \mathrm{C}$, in superior mammals.

With the oscillation set up correctly, prior to reanimation, the issue, on the one hand, would be in the induction of sufficient neuroendocrine secretion, especially in the pineal and hypothalamus and, on the other, in reestablishing heart rate, blood circulation and respiration.

This would first have to be done on the physiologically dead organism. Due techniques of artificial life would be applied while stimulating the pineal gland using an adequate electromagnetic helmet capable of emitting deep-ultraviolet light. The effects of micro-magnetic fields in the pineal gland are started now to be well 
known. On the other hand our unpublished preliminary studies in detained-light and mitochondrial filamentation "in vitro", presented to the First International Congress of Electromagnetism celebrated in Madrid several years ago do suggest the possible utility of such stimulation on pineal cell's filamented mitochondria. This is a mitochondrial character that especially in some pineal cells it is very resistant to low temperatures.

After a few hours in this state, external support aids would be gradually reduced and subsequently small, sharp interruptions would be made to gain autonomous control. The return to a normal life of the superior animal would be demonstrated if it behaved in approximately the same way as before its transitory death. Only then if after obtaining full successful trial results on a representative sample of adult apes, could we advance to the human baby.

\section{DISCUSSION}

With the addition of this research proposal, the methods suggested to try to suspend partially or totally the physiological life of complex organisms have a newly arrived seed concept to the human imagination. It is placed in the acute, o semi-chronic, time range. In my opinion to extend the metabolic life for more than two months will need very fatty organisms.

If this research, with the decades, will prove to be useful, perhaps it will be later surpassed by unknown, at present effective methods, of provoking chronic partial suspension of metabolism for months or years at very low temperatures. Thus is to say "Artificial Hibernation”. In my modest opinion such methods are now far out of reach.

It could be argued really many things, I am well aware, working against the probability that our present sciences could obtain in corpses a well all spread throughout all the organism of metabolic activity extension for too long times. In my opinion our first trials, until the methodologies were well perfected, must not intent physiological death for most that 3 days, in animals, and one day in sudden death babies when if it were possible to arrive as a first end.

Let me suggest please, that this research proposal could be considered as a first real international project, in spite that some of the great scientific potencies of the world could carry it all by them alone. An accrual of research institutions, independently to the task, with insufficient capability and concatenation, will yield surely early macabre failures that could end hopes or give a long retardation to a brighter future. By world science true coordination duly passing to the human baby when absolutely all would be very well controlled in high order primates.

\section{CONCLUSIONS}

In the long term, the problems of this new endeavor I am postulating need to be articulated now, calmly and systematically, with the help of everyone; there will be numerous ones.

On obtaining the first successes, which I suspect may come quite quickly using low order mammals and short times of physiological death, many problems can be foreseen whether matters are handled properly, such as, blood dyscrasias and the loss of teeth, nails and hair.

The more complex subjects may require time to adapt to their new life. However, I would expect that deep memory will not be lost and the capability to gradually piece the substance of the previous life together will be recovered. In short, as for any new adventure of ideas, bringing matters to a successful conclusion and in a timely manner will fall, above all, on the younger generation. Enough time should be given, please, generously.

It only remains for me to proffer advice on filamented mitochondria, and that is for just a few more years until I am overwhelmed by the new experts. Of course, I will also ask for prudence and patience. Plus, of course, a great care in all the experimental treatments: Of what could result some day, in the more or less distant future, transitory cadavers?

\section{ACKNOWLEDGEMENTS}

The author is very grateful to his personal friend, Mr. Andrew Guy, for the translation of the text from Castilian Spanish into English.

\section{REFERENCES}

[1] M. Gosalvez, "Extension of Metabolic Life?” 36th ISOTT Meeting, Sapporo, 3-7 August 2008.

[2] M. Gosalvez, "Mitochondrial Filamentation: Some Methods of Isolation and Assay," IOSR Journal of Pharmacy and Biological Sciences, Vol. 4, No. 4, 2012, pp. 237-239.

[3] M. Gosalvez, "Metabolic Control of Respiration and Glycolysis of Tumoral Cells," Advances in Biological Chemistry, Vol. 3, No. 1, 2013, pp. 86-89.

http://dx.doi.org/10.4236/abc.2013.31011

[4] M. Gosalvez, "Methods to Be Developed for Some First Applications of Mitochondrial Filamentation,” Open Journal of Biophysics, Vol. 3, 2013, pp. 51-53. http://dx.doi.org/10.4236/ojbiphy.2013.31A006

[5] M. Gosalvez, "Reversal of Cancer by Dual Strategy?" Journal of Cancer Therapy, Vol. 4, No. 2, 2013, pp. 518520. http://dx.doi.org/10.4236/jct.2013.42064

[6] M. Gosalvez, "Cancer as a Therapeutic Agent? An Experimental Proposal in Low Order Laboratory Animals," Journal of Cancer Therapy, Vol. 4, 2013, pp. 1362-1365. http://dx.doi.org/10.4236/jct.2013.48161

[7] M. Gosalvez, "Mitochondrial Filamentation: A Thera- 
peutic Target for Neurodegeneration and Aging,” American Journal of Alzheimer and Other Dementias, Vol. 28, No. 5, 2013, pp. 423-426.

http://dx.doi.org/10.1177/1533317513494451
[8] M. Gosalvez, "The Grand Planetary Oxygen Cycle? A Whole Perspective,” Online Journal of Earth Sciences, 2013, in Press. 